

\title{
A STEADY-STATE APPROACH TO PREVENT VOLTAGE COLLAPSE IN POWER SYSTEMS
}

\author{
Jairo Quirós Tortós \\ Eddie Araya Padilla
}

\begin{abstract}
This paper presents a steady state approach to prevent voltage collapse in a power system. The power system operation close to stability boundaries is an important factor which may lead to voltage instability conditions. There are different ways to prevent voltage collapse in the power system; one of this is the correct coordination of the protection devices to avoid undesirable equipment disconnections. This paper proposes a new method to coordinate the back-up zone in the distance protection scheme. The methodology uses both Optimal Power Flow (OPF) and Continuation Power Flow (CPF) solutions to determine the impedance seen by the relay. These impedances are later compared to conclude which is the best option to set the parameter for the back-up zone in the relay. The methodology also considers the distance to Maximum Loading Point (MLP) and the corresponding Voltage Collapse Point (VCP). Therefore, undesirable disconnections can be avoided when the power system operates close to the stability boundaries. In order to demonstrate and validate the methodology, simulations are carried out on the IEEE 14-bus test system using MATLAB.
\end{abstract}

Keywords: Distance protection, optimal power flow, voltage collapse.

\section{Resumen}

Este artículo presenta un enfoque en régimen permanente para prevenir el colapso de tensión en un sistema de potencia. La operación del sistema de potencia cercana a los límites de estabilidad es un importante factor que puede conllevar a condiciones de inestabilidad de tensión. Existen diferentes formas de prevenir el colapso de tensión en el sistema de potencia, la correcta coordinación de los dispositivos de protección de modo tal que se evite la indeseada operación de los equipos es una alternativa. Este artículo propone un nuevo método para coordinar la zona de respaldo en el esquema de protección de distancia. La metodología utiliza dos tipos de soluciones, la solución del flujo optimo de potencia (OPF, por sus siglas en inglés) y la solución del flujo de potencia de continuación (CPF, por sus siglas en inglés) para determinar la impedancia vista por el relé. Estas impedancias son luego comparadas para concluir cuál es la mejor opción para ajustar la zona de respaldo del relé de protección. La metodología también considera la distancia al máximo punto de cargabilidad (MLP, por sus siglas en inglés) y el correspondiente punto de colapso de tensión (VCP, por sus siglas en inglés). Para demostrar y validar la metodología, simulaciones son llevadas a cabo en el sistema de prueba IEEE-14 barras utilizando MATLAB.

Palabras clave: Protección de distancia, flujo óptimo de potencia, colapso de tensión.

Received: 6 of April, 2012 Aproved: 14 of May, 2013

\section{INTRODUCTION}

The continuous changes in the power system topology to meet the expansion plans and the interconnections with other countries have caused an increase in the number of power system blackouts. The impact of these blackouts in the economy has encouraged more researchers to determine different methods to avoid these undesirable events. By properly setting the protection devices, undesirable cascading outages can be avoided.

Voltage stability can be described as the capability of the system to maintain their bus voltage magnitudes within a permissible operating threshold under normal operating conditions 
and after the disturbances arise (Kundur, 1994). Massive system interconnections, increased demands, insufficient generation, transmission expansion, economical and environmental factors have led power systems to operate very close to their limits.

In real power systems, the experiences of voltage collapse are massive. In (Jonsson, 2003; Ohno \& Imai, 2006; Ruiz, 2006; Sancho \& Vega, 2001) the authors describe what happened in the power system causing the voltage collapse. Common characteristics have been identified when a voltage collapse happens. Some of these common features are:

- Slow dynamic behaviour: Operation of On-Load Tap Changers (OLTC) and over excitation current limiters in generators.

- The decrease in the voltage magnitude at buses more than expected.

- The disconnection of the faulted line by a conventional relay: distance relay or differential protection scheme.

- Undesirable trip out of important transmission lines and sources of reactive power.

Voltage stability studies have been typically carried out using static approaches such as power flow analysis (Gan, Thomas, \& Zimmerman, 2000; Momoh \& Shu, 1999; Vaahedi, Tamby, Manssur, Li, \& Sun, 1999), P-V curves (Johansson \& Sjögren, 1995; Jonsson, 2003; Kundur, 1994; Nagao, Tanaka, \& Takenaka, 1997; Rajagopalan, Lesieutre, Sauer, \& Pai, 1992; Repo, 2001; Taylor, 1994), V-Q curves (Chowdhury \& Taylor, 2000; Johansson \& Sjögren, 1995; Jonsson, 2003; Kundur, 1994; Overbye, Dobson, \& DeMarco, 1994; Taylor, 1994), modal analysis (Gao, Morison, \& Kundur, 1992; Morison, Gao, \& Kundur, 1993), and others techniques including continuation power flow techniques and the correspondent $\lambda-\mathrm{V}$ curves (Alinezhad \& Kamarposhti, 2009; Milano, 2005; Venkataramana, 2006; Venkataramana \& Christy, 1992). More complex methods including detailed and realistic models of power system elements and their controllers are considered on dynamic simulations which can be effectively simplified using the so-called Quasi Steady State Simulation (Van Cutsem \& Vournas, 1998).
This paper presents a methodology to determine the settings for the back-up zone in the distance protection scheme to avoid voltage collapse in a power system. By using the $\lambda-\mathrm{V}$ curve, obtained from the CPF algorithm (Milano, 2005; Venkataramana, 2006; Venkataramana \& Christy, 1992), the distance to the VCP can be easily determined as the distance in megawatts between the actual operating point and the bifurcation point (nozzle of the curve). At this point, the corresponding electrical variables are determined to compute the settings for the backup zone (generally third zone) in the distance protection scheme.

When a system is subject to a severe disturbance, depending on the loading conditions of the system, it can move the operating point of the system into a condition where voltages in parts of the system are below the permissible limits. In this sense, part of the system will face an unstable condition which might cause undesirable operation of protective devices. By this, unexpected disconnection of reactive power sources accelerates the voltage collapse (Van Cutsem \& Vournas, 1998). To avoid system blackout caused by mal-operation of relays, it is necessary to make a correct setting of the protection devices, which is the main goal of this paper.

This paper is organised as follow. The continuation power flow algorithm and the optimal power flow algorithm are presented in Section 2 and Section 3, respectively. The proposed methodology to coordinate the distance protection scheme is then presented in Section 4. Simulations are carried out in Section 5 to demonstrate and validate the proposed method. Final remarks are given in Section 6.

\section{CONTINUATION POWER FLOW ALGORITHM}

The continuation power flow is an important tool to determine the power flow in a power system starting from a base case and leading to the steady state voltage stability limit of the system (Venkataramana, 2006; Venkataramana \& Christy, 1992). Therefore, this algorithm is used worldwide to determine the power flow solution when the system operates close to the VCP. 
The employed algorithm avoid the singularity of the Jacobian by reformulating the power flow equations and applying locally parameterized continuation technique (Venkataramana, 2006; Venkataramana \& Christy, 1992).

As it can be noticed in Figure 1, the CPF determines the power flow solution for a base case first. Then, a tangent predictor is used to estimate the next solution corresponding to a different value of the load parameter. This estimate is then corrected using the same Newton-Raphson algorithm employed by a conventional power flow. The local parameterization previously mentioned provides a mean of identifying each point along the solution path and plays an integral part in avoiding singularity in the Jacobian (Venkataramana, 2006; Venkataramana \& Christy, 1992).

Based on the original nonlinear power system equations, $F(\underline{x})=0$, where $\underline{x}=[\underline{v}, \underline{\theta}]$, the $\mathrm{CPF}$ formulation introduces a load parameter $\lambda$ to apply a locally parameterised continuation technique to the power flow problem. Therefore, the new set of nonlinear power system equations is represented by:

$F(x, \lambda)=0$

This parameter $\lambda$, which varies from 0 up to a critical value i.e. $0 \leq \lambda \leq \lambda$ critical, is used to reformulate the power flow equations in a power system with $N_{b}$ buses. Therefore, if $\underline{F}$ is used to denote the whole set of equations, the problem can be expressed as follow:

$\underline{F}(\underline{v}, \underline{\theta}, \lambda)=0 \quad 0 \leq \lambda \leq \lambda_{\text {critical }}$

Where $\underline{v}$ and $\underline{\theta}$ represent the vector of bus voltage magnitudes and phases. Here, $\lambda=0$ represents the base load and $\lambda=\lambda_{\text {critical }}$ corresponds to the critical load. As it was previously mentioned, the base case solution $\left(\underline{v}_{0}, \underline{\theta}_{0}, \underline{\lambda}_{0}\right)$ is known via a conventional power flow and the solution path is being sought over a range of $\lambda$.

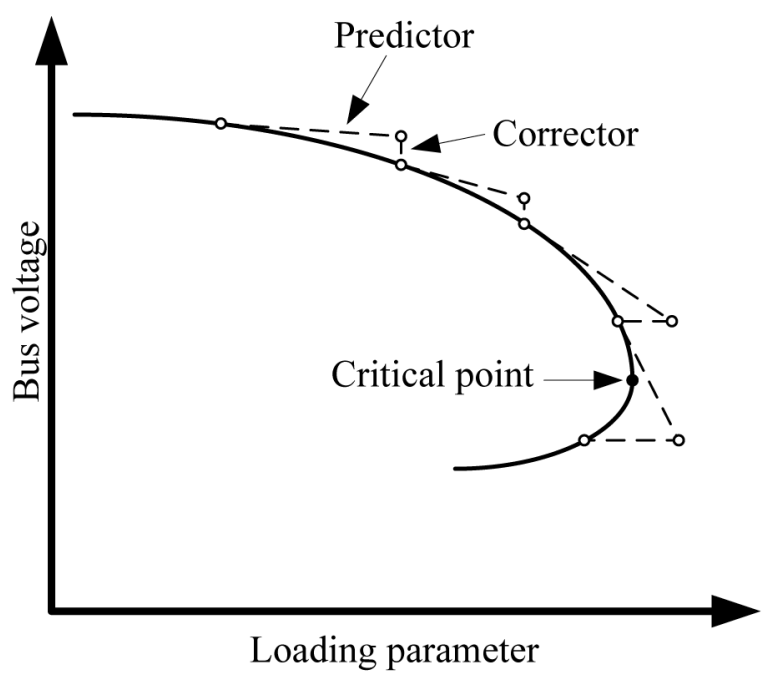

Figure 1. Predictor-corrector scheme used in the CPF. Source: Venkataramana \& Christy, 1992. 
These set of equations can be expressed in terms of the power load, power generation and power injection at each bus $i$ as follows:

$$
\begin{aligned}
& P_{i}(v, \theta, \lambda)=P_{i}(v, \theta)+P_{d, i}(\lambda)-P_{g, i}(\lambda)=0 \\
& Q_{i}(v, \theta, \lambda)=Q_{i}(v, \theta)+Q_{d, i}(\lambda)-Q_{g, i}=0
\end{aligned}
$$

where, (Equations 5 and 6)

and $\mathrm{P}_{d, i}(\lambda)$ and $Q_{d, i}(\lambda)$ are the active and reactive power load at the bus $i$, respectively, $\mathrm{P}_{g, i}(\lambda)$ and $Q_{g, i}$ are the active and reactive power generation at the bus $i$, respectively. In (5) and (6), $\mathrm{G}_{i j}$ is the $i j$-th element of the bus conductance matrix $\mathrm{G}, \mathrm{B}_{i j}$ is the $i j$-th element of the bus susceptance matrix $\mathrm{B}, v_{i}$ and $\theta_{i}$ are the bus voltage magnitude and phase at bus $i$.

To simulate the load change, $\mathrm{P}_{d, i}(\lambda)$ and $Q_{d, i}$ terms are modified as follows:

$$
\begin{aligned}
& P_{d, i}(\lambda)=P_{d, i, 0}\left[1+\lambda K_{d, i}\right] \\
& Q_{d, i}(\lambda)=Q_{d, i, 0}\left[1+\lambda K_{d, i}\right]
\end{aligned}
$$

where $P_{d, i, 0}$ and $Q_{d, i, 0}$ are the original active and reactive power load at bus $i$, respectively, and $K_{d, i}$ is a multiplier to designate the rate of load change at bus $i$ as $\lambda$ changes.

Similar to the power load, power generation is also modified as follow:

$$
P_{g, i}(\lambda)=P_{g, i, 0}\left[1+\lambda K_{g, i}\right]
$$

where $P_{g, i, O}$ is the active power generation at bus $i$ in the base case and $K_{g, i}$ is a constant used to specify the rate of generation change at bus $i$ as $\lambda$ changes.

When the base case solution is obtained, the prediction of the next solution is made by taking an appropriated size step in a direction tangent to the solution path. Thus, the tangent vector is obtained by taking the derivative of both sides of the power flow equations.

$$
d[\underline{F}(\underline{v}, \underline{\theta}, \lambda)]=\underline{F}_{v} d \underline{v}+\underline{F}_{\theta} d \underline{\theta}+\underline{F}_{\lambda} d \lambda=0
$$

Factorizing,

$$
\left[\begin{array}{lll}
\underline{F}_{v} & \underline{F}_{\theta} & \underline{F}_{\lambda}
\end{array}\right]\left[\begin{array}{l}
d \underline{v} \\
d \underline{\theta} \\
d \lambda
\end{array}\right]=0
$$

As it can be noticed in (11), the matrix of partial derivative $\left[\begin{array}{lll}\underline{F}_{v} & \underline{F}_{\theta} & \underline{F}_{\lambda}\end{array}\right]$ represent the conventional power flow Jacobian augmented by one column $\left(\underline{F}_{\lambda}\right)$, while the vector of differentials $[d \underline{v} d \underline{\theta} \mathrm{d} \lambda]^{T}$ is the vector being sought.

At this point, it is important to visualise that as one additional unknown was added when $\lambda$ was inserted into the power flow equations, but the number of equations remained unchanged, and, therefore, one more equation is now needed. This problem is solved by choosing a non-zero magnitude for one of the components of the tangent vector.

$$
\underline{r}=\left[\begin{array}{l}
d \underline{v} \\
d \underline{\theta} \\
d \lambda
\end{array}\right] \quad r_{k}= \pm 1
$$

Where $\underline{r}$ represents the tangent vector with a corresponding dimension $c=2 n_{1}+n_{2}+1\left(n_{1}\right.$ and $n_{2}$ are the number of load and generation buses, respectively). This results in equation 13 .

$$
\begin{aligned}
& P_{i}(v, \theta)=v_{i}^{2} G_{i i}+\sum_{\substack{n=1 \\
n \neq i}}^{N_{b}}\left[v_{i} v_{n} G_{i n} \cos \left(\theta_{i}-\theta_{n}\right)+v_{i} v_{n} G_{i n} \sin \left(\theta_{i}-\theta_{n}\right)\right] \\
& Q_{i}(v, \theta)=-v_{i}^{2} B_{i i}-\sum_{\substack{n=1 \\
n \neq i}}^{N_{b}}\left[v_{i} v_{n} B_{i n} \cos \left(\theta_{i}-\theta_{n}\right)-v_{i} v_{n} B_{i n} \sin \left(\theta_{i}-\theta_{n}\right)\right]
\end{aligned}
$$


$\left[\begin{array}{ccc}\underline{F}_{v} & \underline{F}_{\theta} & \underline{F}_{\lambda} \\ & \underline{e}_{k} & \end{array}\right][\underline{r}]=\left[\begin{array}{c}0 \\ \pm 1\end{array}\right]$

where $\underline{e}_{k}$ is a row vector with all elements equal to zero except the $k$-th, which is equal to one. When the tangent vector is obtained by solving (13), the prediction can be made as follows:

$$
\left[\begin{array}{l}
\underline{v}^{l+1} \\
\underline{\theta}^{l+1} \\
\lambda^{l+1}
\end{array}\right]=\left[\begin{array}{l}
\underline{v}^{l} \\
\underline{\theta}^{l} \\
\lambda^{l}
\end{array}\right]+\sigma\left[\begin{array}{l}
d \underline{v} \\
d \underline{\theta} \\
d \lambda
\end{array}\right]
$$

Where $l+1$ represents the predicted solution for a subsequent value of $\lambda$, and $\sigma$ is a scalar that designated the step size. This step size should be selected so that the predicted solution is within the radius of convergence of the corrector. When the prediction is made, the corrector is developed. Local parameterization is used in this paper, thus, the reformulated power flow equations (2) are expressed as follow:

$$
\left[\begin{array}{c}
F(\underline{x}) \\
x_{k}-\eta
\end{array}\right]=0 \quad \underline{x}=\left[\begin{array}{l}
\underline{v} \\
\underline{\theta} \\
\lambda
\end{array}\right]
$$

Where $\eta$ is an appropriate value for the $k$-th element of $\underline{x}$.

The continuation parameter is selected at each step based on the state variable with the largest tangent vector component. The load parameter $\lambda$ is a good guess for power systems. Then, equation is used to calculate $x_{k}$, the continuation parameter.

$$
x_{k}:\left|r_{k}\right|=\max \left\{\left|r_{1}\right|,\left|r_{2}\right|, \ldots,\left|r_{m}\right|\right\}
$$

Where $k$ corresponds to the component of the tangent vector that is maximal. As the VCP represented the point at which $d \lambda=0$, by computing $d \lambda$ and by monitoring when this value changes from positive to negative the VCP is determined.

\section{OPTIMAL POWER FLOW ALGORITHM}

The optimal power flow algorithm uses optimization techniques to find the optimal state of a power system while satisfying a set of physical and operational constraints (Momoh \& Shu, 1999). Most OPF problems can be represented as follows:

minimize $f(\underline{x})$

subject to

$$
\begin{array}{r}
g(\underline{x})=0 \\
\underline{h}_{\text {min }} \leq h(\underline{x}) \leq \underline{h}_{\max } \\
\underline{x}_{\min } \leq \hat{I} \underline{x} \leq \underline{x}_{\max }
\end{array}
$$

Where $\underline{x}$ is the vector of explicit decision variables, $f(\underline{x})$ is a scalar function representing the power system's planning or operation optimization goal, $g(\underline{x})$ is a nonlinear vector containing the equality constraints on the system, $h(\underline{x})$ is a nonlinear vector of functional variables with lower bound $\underline{h}_{\min }$ and upper bound $\underline{h}_{\max }$ representing specific operational limits on the system. $\hat{I} \underline{x}$ is a vector with components of $\underline{x}$ that have finite bounds (lower bound $\underline{x}_{\min }$ and upper bound $\underline{x}_{\max }$ ) corresponding to physical and specified operational limits on the system, $\hat{I}$ is an incidence matrix to obtain $\hat{I} \underline{x}$ from $\underline{x}$. It is assumed that $f(\underline{x}), g(\underline{x})$ and $h(\underline{x})$ are continuous and differentiable at least twice for $\underline{x}$. In this paper, $n$ number of primal variables $x_{i}, m$ number of equality constraints, $p$ number of nonlinear functional bound constraints, and $q$ number of simple bound constraints are considered.

Similar to the CPF, and as the equality constraints, the OPF algorithm considered a double set of $N_{b}$, nonlinear active and reactive power balance equations at each bus $i$. These are formulated similarly as the CPF algorithm, but considering $\lambda=0$, this is:

$$
\begin{aligned}
& g_{P, i}(v, \theta)=P_{i}(v, \theta)+P_{d, i}-P_{g, i} \\
& g_{Q, i}(v, \theta)=Q_{i}(v, \theta)+Q_{d, i}-Q_{g, i}
\end{aligned}
$$


Where the active and reactive power injections at bus $i$ are computed by using (5) and (6), respectively. $P_{d, i}$ and $Q_{d, i}$ are the active and reactive power load at bus $i$, respectively. $P_{g, i}$ and $Q_{g, i}$ are the active and reactive power generation at bus $i$, respectively.

The inequality constraints are formed of two sets of $N_{l}$, where $N_{l}$ represents the number of branches in the power system, branch flow limits as nonlinear functions of the bus voltage magnitudes and phases, one for the sending end $i$ and one for the receiving end $j$ of each branch.

$h_{i j}(v, \theta, t)=\left|P_{i j}(v, \theta, t)+j Q_{i j}(v, \theta, t)\right|-F_{\max } \leq 0$

$h_{j i}(v, \theta, t)=\left|P_{j i}(v, \theta, t)+j Q_{j i}(v, \theta, t)\right|-F_{\max } \leq 0$

Where the power flow in branches is computed by considering the tap setting representation and the $\pi$-model of the transmission line as follows:

(Equations 22 and 23)

In (22) and (23), $t_{i j}, g_{i j}, b_{i j}$, and $b_{i j}^{\text {sh }}$ are the tap setting, the series conductance, the series susceptance and the shunt susceptance of the branch $(i, j)$, respectively. In these equations, $t_{i j}=1$ if the branch $(i, j)$ is a transmission line and $\mathrm{b}_{i j} \mathrm{sh}=0$ if branch $(i, j)$ is a transformer.

In the algorithm, the vector of explicit decision variables $\underline{x}$ is represented by the vector of voltage magnitudes $\underline{v}$, the vector of voltage phases $\underline{\theta}$ (the reference bus is defined as $\theta_{1}=0^{\circ}$ ), the vector transformer tap settings $\underline{t}$, and the vectors of active $\left(\underline{P}_{g}\right)$ and reactive $\left(Q_{g}\right)$ power generations as it can be noticed in (24).

$$
\underline{x}=\left[\begin{array}{c}
\underline{v} \\
\underline{\theta} \\
\underline{t} \\
\underline{P}_{g} \\
\underline{Q}_{g}
\end{array}\right]
$$

Each bus, tap setting, and generators would include a double set of finite bounds which is represented as follows:

$\begin{array}{ll}\theta_{i, \text { min }} \leq \theta_{i} \leq \theta_{i, \max } & \text { for all buses } \\ v_{i, \text { min }} \leq v_{i} \leq v_{i, \text { max }} & \text { for all buses } \\ t_{i j, \min } \leq t_{i j} \leq t_{i j, \text { max }} & \text { for all transformers } \\ P_{i, \text { min }} \leq P_{i} \leq P_{i, \text { max }} & \text { for all generators } \\ Q_{i, \text { min }} \leq Q_{i} \leq Q_{i, \max } & \text { for all generators }\end{array}$

As voltage stability problems are under studied, the system loss for a given load and generation represents the objective function in the proposed OPF formulation. This is a function of the reactive power flow, which is mainly dictated by the system node voltage magnitudes, as the variation in the node phases calculated in the initial load flow is small.

$$
\min F=\min \sum_{i=1}^{N_{h}} \sum_{j=1}^{N_{j}} G_{i j}\left(v_{i}^{2}+v_{j}^{2}-2 v_{i} v_{j} \cos \left(\theta_{i}-\theta_{j}\right)\right)
$$

\section{DISTANCE PROTECTION COORDINATION TO AVOID VOLTAGE COLLAPSE}

High voltage transmission lines are commonly protected by distance protection schemes (Jonsson, 2003; Jonsson \& Daalder, 2003; Yang, Shi, \& X., 2006). The main objective of the protection devices is to clear the fault and isolate the faulted transmission line as quickly as possible considering the critical clearing time previously determined by grid codes. On the other hand, voltage collapse sometimes occurs after undesirable trip of the transmission lines (Johansson \& Sjögren, 1995; Jonsson, 2001, 2003; Ohno \& Imai, 2006; Sancho $\&$ Vega, 2001). Thus, the correct coordination of the distance protections to reduce the number of undesirable trips is vital in current power systems.

$$
\begin{aligned}
& P_{i j}(v, \theta, t)=t_{i j}^{2} g_{i j} v_{i}^{2}-t_{i j} v_{i} v_{j}\left[g_{i j} \cos \left(\theta_{i}-\theta_{j}\right)+b_{i j} \sin \left(\theta_{i}-\theta_{j}\right)\right] \\
& Q_{i j}(v, \theta, t)=-t_{i j}^{2}\left(b_{i j}+b_{i j}^{s h}\right) v_{i}^{2}-t_{i j} v_{i} v_{j}\left[g_{i j} \sin \left(\theta_{i}-\theta_{j}\right)-b_{i j} \cos \left(\theta_{i}-\theta_{j}\right)\right]
\end{aligned}
$$


The third zone in the distance protection scheme has been used as a back-up zone (Abba-Aliyu, 2009; Horowits \& Phadke, 2006; Jonsson \& Daalder, 2003). Therefore, the incorrect setting in this zone might produce an undesirable disconnection of the transmission line during voltage instability, accelerating the process of blackout.

As the voltage instability is a symmetric phenomenon, i.e. only positive sequence components are involved (Jonsson, 2003), the impedance seen by the relay can be calculated as follow.

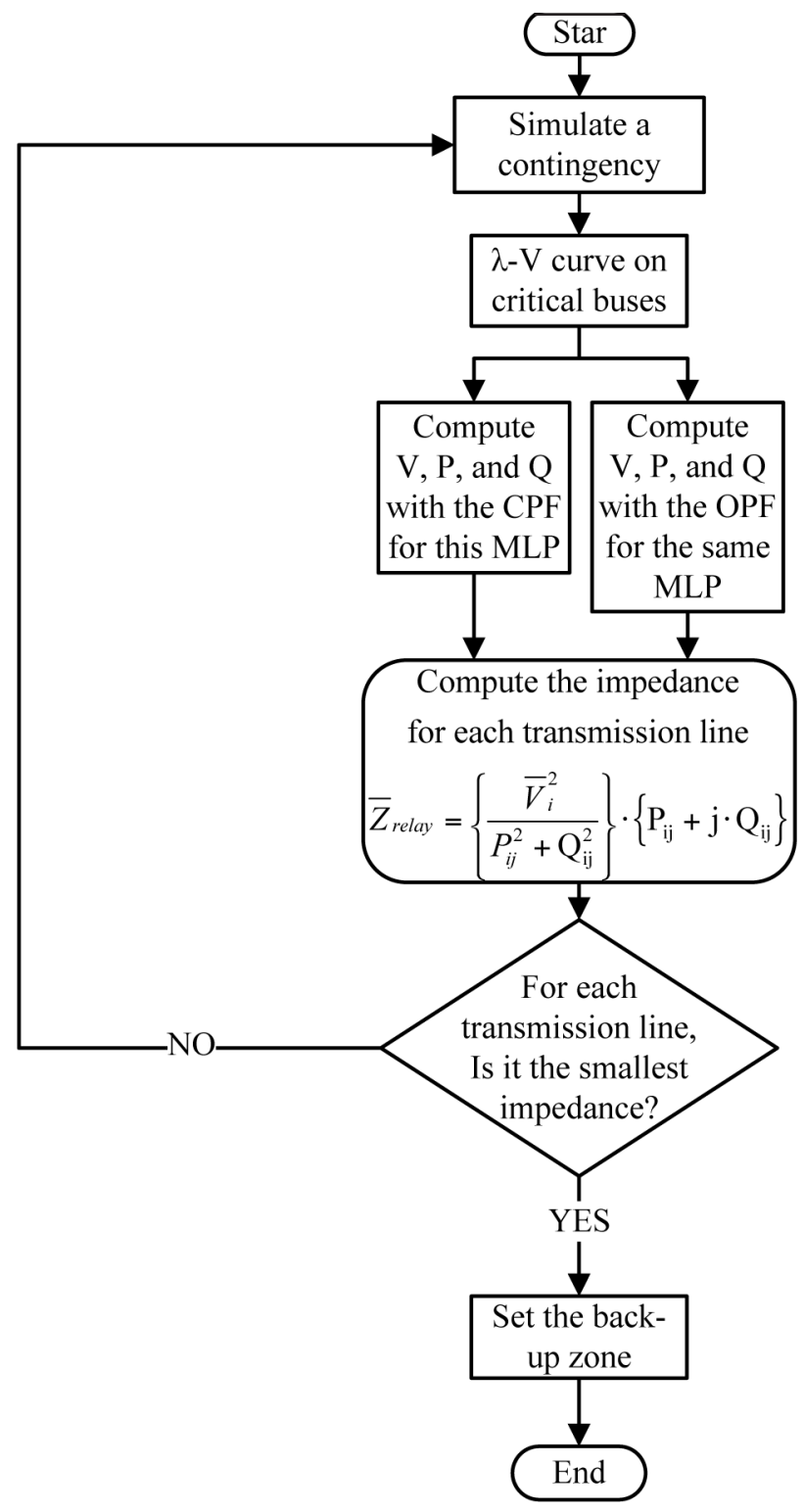

Figure 2. Flowchart for correct coordination of the distance protection to avoid voltage collapse. 


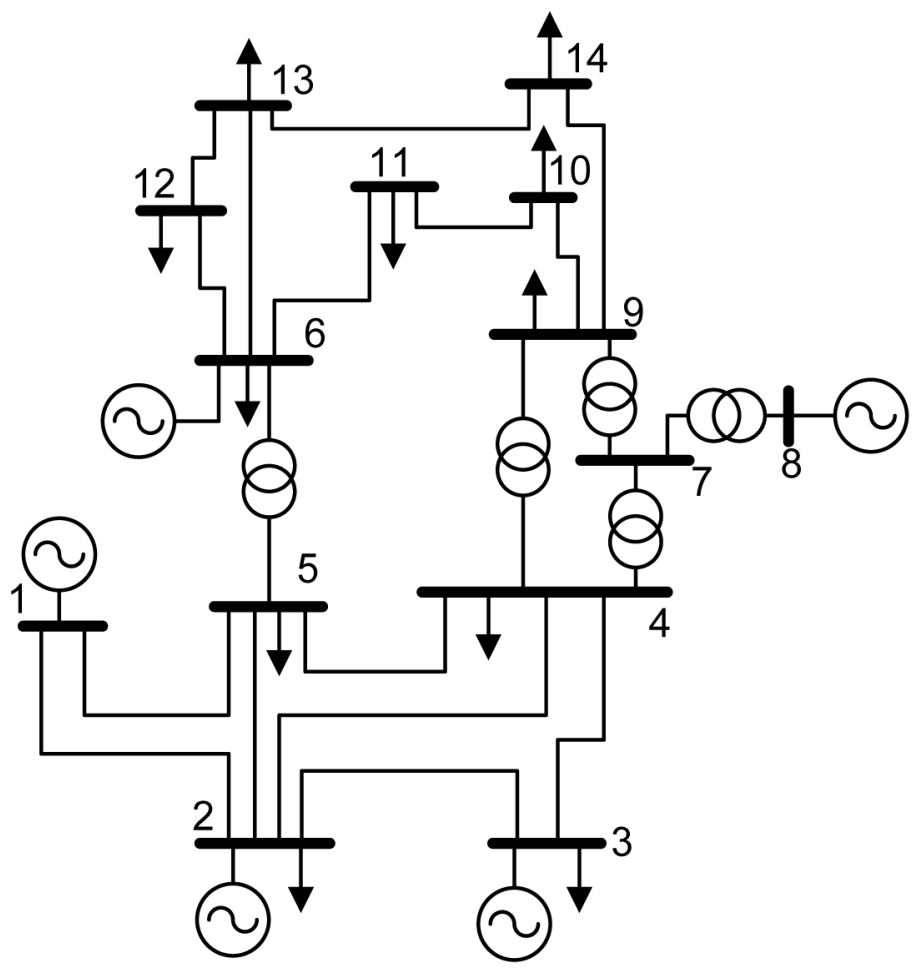

Figure 3. Single line diagram of the IEEE 14- bus test system. Source: University of Washington, 2010.

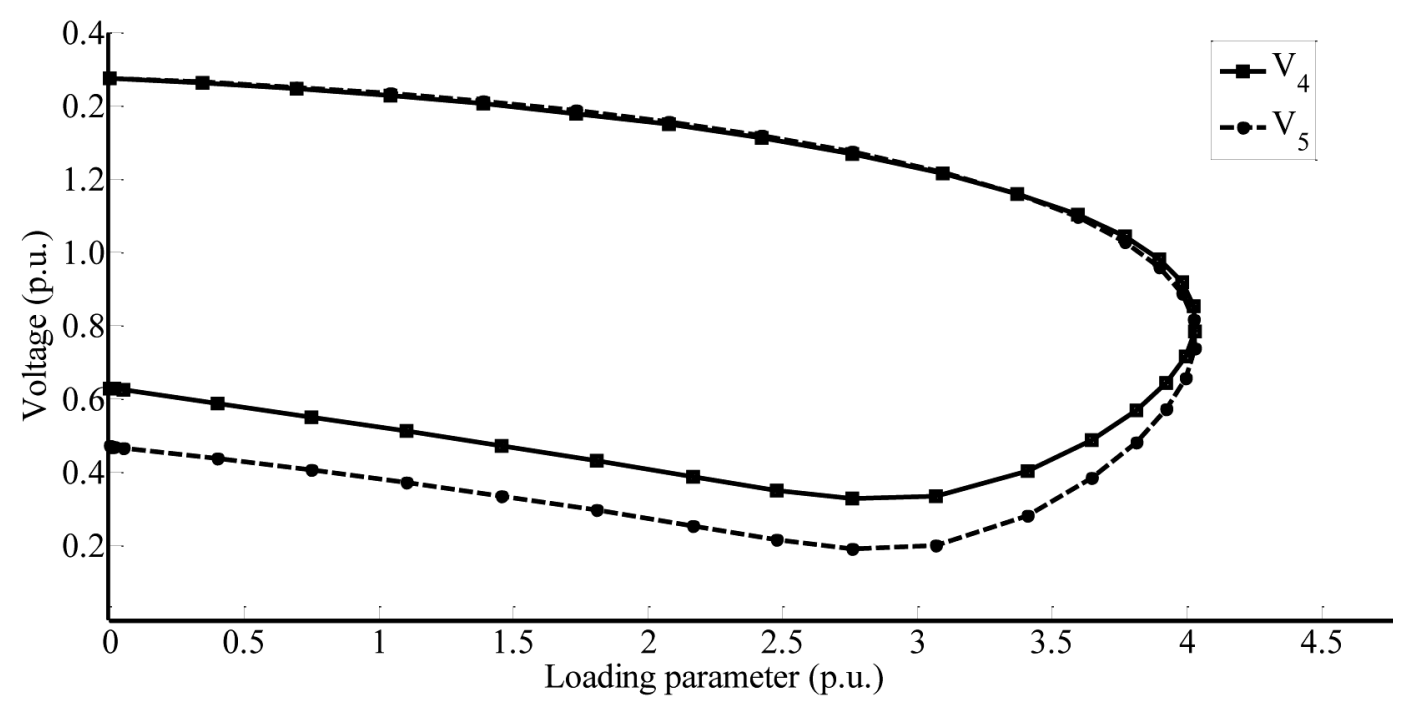

Figure 4. $\lambda-V$ curve for base case on bus 4 and bus 5 .

Source: Authors, 2012. 


$$
\bar{Z}_{\text {relay }}=\frac{\bar{v}_{i}^{1}}{\bar{I}_{i j}^{1}}
$$

Where $\overline{\mathrm{v}}_{i}^{l}$ is the phasor of positive sequence voltage at the relay location and $\overline{\mathrm{I}}_{i j}^{l}$ is the phasor of positive sequence current flowing from bus $i$ to bus $j$. Equation (27) can be also expressed in terms of the transferred complex power as follow.

$$
\bar{Z}_{\text {relay }}=\frac{\left|\bar{v}_{i}^{1}\right|^{2}}{\bar{S}_{i j}^{*}}
$$

Or:

$$
\bar{Z}_{\text {relay }}=\frac{\left|\bar{v}_{i}^{1}\right|^{2}}{P_{i j}^{2}+Q_{i j}^{2}}\left(P_{i j}+j Q_{i j}\right)
$$

According to (29), if the voltage at the relay location and power in the transmission line, which is protected by the relay, are known, it is possible to determine the impedance to set the protection device in order to avoid line disconnection under voltage instability conditions.

The methodology to determine the smallest impedance is shown in Figure 2. This method is iteratively carried out for the each considered contingency. In this figure, the $\lambda-\mathrm{V}$ curve is obtained for those critical buses to determine the critical point as previously shown in Figure 1. When the MLP is known, the CPF and the OPF algorithms are run for a load condition around this MLP. The electrical variables, voltage, current, and power flow, are then saved to compute the impedance according to each method. When these impedances to set the back-up zone are known, a direct comparison of both methods is carried out to select the smallest impedance.

\section{SIMULATION RESULTS}

In order to demonstrate and validate the proposed methodology, simulations are carried out on the IEEE 14-bus test system University of Washington, 2010. The topology of this test system is shown in Figure 3. The IEEE 14-bus has 5 generating units with bus
1 chosen as the slack bus. The system includes 16 transmission lines, 4 transformers and 11 load buses. The system has 7 transmission lines in the high voltage side. Because of this, the system has 14 different distance relays, each one in both sides of the high voltage transmission lines.

\subsection{Continuation power flow results}

Using the CPF algorithm, the $\lambda$-V curves are built for critical buses. Figure 4 shows the $\lambda$-V curve at bus 4 and bus 5 for the case when no contingency is considered. As it can be calculated from Figure 4, the critical point is reach when $\lambda_{\text {critical }}=4.0303$ p.u.

Different contingencies in the high voltage level, where distance protections are placed, are considered to analyse the system response. For every contingency, the voltage, active, and reactive power in the transmission lines are computed. Then, by using the proposed methodology, the smallest impedance for each side of the transmission line can be determined. These calculated impedances are shown in Table 1. This impedance (in per unit) will be then used to adjust the impedance setting for the back-up zone to avoid undesirable transmission line disconnections.

Analysing all contingencies, two critical scenaries are identified. The first one happens when the transmission line 1-5 is disconnected and the second one occurs with the outage of transmission line 4-5. For these two cases, important reduction of the voltage levels and high power flows through the transmission lines are obtained. Figure 5 and 6 show the $\lambda-\mathrm{V}$ curves at bus 4 and bus but considering transmission lines 1-5 and 4-5 disconnected, respectively. As it can be noticed, the maximum loading point for these cases are obtained when $\lambda_{\text {critical }}=3.6539$ p.u. and when $\lambda_{\text {critical }}=3.9348$ p.u., respectively.

\subsection{Optimal power flow results}

Similar simulations are carried out for all outages considered in Table 1 but using the OPF algorithm presented in Section 3. By minimising the power losses, the reactive sources are improved and, therefore, the voltage stability margins are enhanced. 
Table 1. Results from CPF simulations to coordinate distance protection to avoid voltage collapse

\begin{tabular}{ccccccccc}
\hline $\begin{array}{c}\text { Transmission } \\
\text { line to set }\end{array}$ & $\begin{array}{c}\text { Voltage at } \\
\text { sending } \\
\text { end }\end{array}$ & $\begin{array}{c}\text { Active power } \\
\text { from sending } \\
\text { end }\end{array}$ & $\begin{array}{c}\text { Reactive } \\
\text { power from } \\
\text { sending end }\end{array}$ & $\begin{array}{c}\text { Impedance } \\
\text { to adjust at } \\
\text { relay }\end{array}$ & $\begin{array}{c}\text { Transmission line } \\
\text { disconnected }\end{array}$ & $\lambda_{\text {critical }}$ \\
bus & $\begin{array}{c}\text { To } \\
\text { bus }\end{array}$ & (p.u.) & (p.u.) & (p.u.) & (p.u.) & $\begin{array}{c}\text { From } \\
\text { bus }\end{array}$ & To bus & (p.u.) \\
\hline 2 & 5 & 1.04500 & 3.6590 & 2.2513 & 0.254188762 & 1 & 5 & 3.6539 \\
1 & 2 & 1.06000 & 16.3046 & 2.8973 & 0.067850151 & 1 & 5 & 3.6539 \\
3 & 2 & 1.01000 & -3.9407 & 3.9004 & 0.183981865 & 4 & 5 & 3.9348 \\
3 & 4 & 1.01000 & -2.1197 & 3.7250 & 0.238014223 & 2 & 3 & 2.2502 \\
1 & 5 & 1.06000 & 4.4728 & 2.3835 & 0.221694508 & 1 & 2 & 1.3363 \\
5 & 4 & 0.64228 & 2.6548 & -0.8495 & 0.147995052 & 2 & 4 & 3.2834 \\
2 & 4 & 1.04500 & 4.3656 & 2.6535 & 0.213754996 & 4 & 5 & 3.9348 \\
5 & 2 & 0.67396 & -2.692 & 0.6744 & 0.163673145 & 1 & 5 & 3.6539 \\
2 & 1 & 1.04500 & -11.5716 & 11.4948 & 0.066952277 & 1 & 5 & 3.6539 \\
2 & 3 & 1.04500 & 5.3648 & 2.0534 & 0.190104265 & 4 & 5 & 3.9348 \\
4 & 3 & 0.79809 & 3.3295 & -0.6478 & 0.187783358 & 2 & 3 & 2.2502 \\
5 & 1 & 0.638350 & -2.4917 & 1.2395 & 0.146422899 & 2 & 5 & 3.4209 \\
4 & 5 & 0.67078 & -2.4033 & 1.6426 & 0.154566847 & 2 & 4 & 3.2834 \\
4 & 2 & 0.684750 & -2.9710 & 1.5489 & 0.139943546 & 4 & 5 & 3.9348 \\
\hline
\end{tabular}

Source: Authors, 2012.

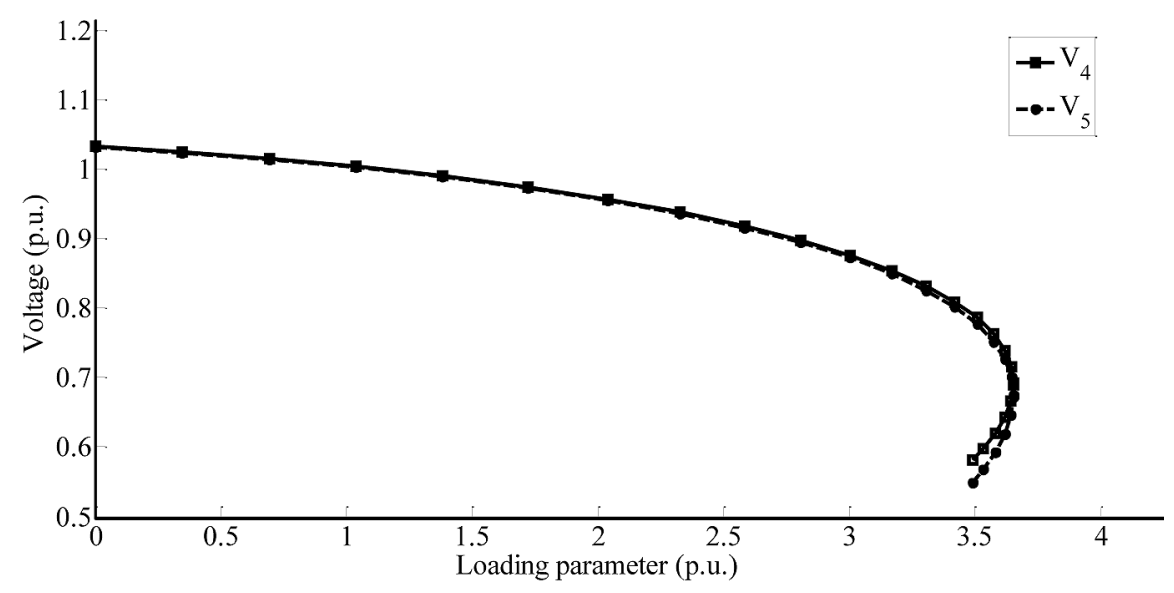

Figure 5. $\lambda-\mathrm{V}$ curve for buses 4 and 5 under the outege of line 1-5. 


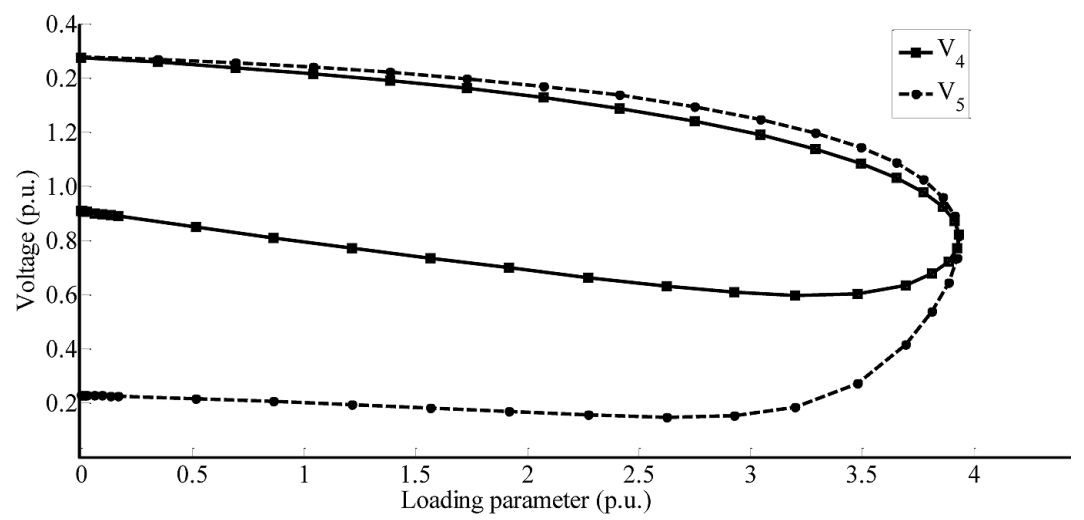

Figure 6. $\lambda-\mathrm{V}$ curve for buses 4 and 5 under the outege of line 4-5.

Source: Authors, 2012.

The simulations using the proposed OPF are carried out for the same operating point as it was obtained from the CPF, i.e. in the MLP. These simulation results are summarized in Table 2. It is necessary to say that when using the OPF, the simulations are not simulated considering exactly the same MLP found by the $\lambda-\mathrm{V}$ curves due to OPF convergence problems when approaching the VCP, therefore, the closest point in this curve is used.
As it is noticed in Table 2, voltage magnitudes are clearly improved i.e. these are closer to 1 p.u. Also, in comparison with the CPF solution, the impedances are larger. By using the OPF algorithm, it is noticed that voltage stability problem is not an issue. In fact, as the constraints included in the formulation consider voltage limits as well as loadability of the branches, the obtained impedances are expected to be larger, as it is noticed in Table 2 .

Table 2. Results from OPF simulations to coordinate distance protection to avoid voltage collapse

\begin{tabular}{|c|c|c|c|c|c|c|}
\hline \multicolumn{2}{|c|}{$\begin{array}{c}\text { Transmission line } \\
\text { to set }\end{array}$} & \multirow{2}{*}{$\begin{array}{c}\text { Voltage at } \\
\text { sending end } \\
\text { (p.u.) }\end{array}$} & \multirow{2}{*}{$\begin{array}{c}\text { Current flow from } \\
\text { sending end } \\
\text { (p.u.) }\end{array}$} & \multirow{2}{*}{$\begin{array}{c}\text { Impedance to } \\
\text { adjust at relay } \\
\text { (p.u.) }\end{array}$} & \multicolumn{2}{|c|}{$\begin{array}{l}\text { Transmission line } \\
\text { disconnected }\end{array}$} \\
\hline From bus & To bus & & & & From bus & To bus \\
\hline 2 & 5 & 1.0462 & 0.58081 & 1.801277526 & 1 & 5 \\
\hline 1 & 2 & 1.1000 & 1.66320 & 0.661375661 & 1 & 5 \\
\hline 3 & 2 & 1.0022 & 0.73952 & 1.355203375 & 4 & 5 \\
\hline 3 & 4 & 0.9366 & 0.79466 & 1.17861727 & 2 & 3 \\
\hline 1 & 5 & 1.1000 & 1.47130 & 0.747638143 & 1 & 2 \\
\hline 5 & 4 & 1.0013 & 0.93834 & 1.067097214 & 2 & 4 \\
\hline 2 & 4 & 1.058 & 0.75266 & 1.405681184 & 4 & 5 \\
\hline 5 & 2 & 0.9716 & 0.59592 & 1.630453752 & 1 & 5 \\
\hline 2 & 1 & 1.0462 & 1.65630 & 0.631648856 & 1 & 5 \\
\hline 2 & 3 & 1.0580 & 0.73286 & 1.443659089 & 4 & 5 \\
\hline 4 & 3 & 0.98974 & 0.79466 & 1.245488637 & 2 & 3 \\
\hline 5 & 1 & 1.0011 & 0.68820 & 1.454664342 & 2 & 5 \\
\hline 4 & 5 & 0.98324 & 0.93834 & 1.047850459 & 2 & 4 \\
\hline 4 & 2 & 0.98436 & 0.76145 & 1.292744107 & 4 & 5 \\
\hline
\end{tabular}




\subsection{Comparison of calculated impedances}

When the impedance of each transmission lines is computed by both algorithms, a direct comparison is carried out. As it is noticed in Table 3, the calculated impedance from the OPF algorithm to set the back-up zone in the protection devices is considerably bigger than the same setting using the CPF method.

This result is due to the considered constraints in the OPF formulation. As it can be noticed in Table 2, the voltage magnitude at each bus is closer to the nominal voltage when using the OPF method. In addition, the OPF method reduces the reactive power losses in the transmission lines. As a consequence, this minimization produce that power flows through the transmission lines are smaller than the power flows obtained from the CPF method.

As it can be noticed in Table 3, the correct settings to avoid an undesirable trip of the distance protections are obtained by the CPF. Table 4 shows the final settings in distance protection to avoid voltage collapse in IEEE 14-bus test system.

\section{CONCLUSION}

A new methodology to coordinate the backup zone in the distance protection devices using the two algorithms was proposed in this paper. Different contingencies were considered and the parameters to set the protection were calculated for both algorithms. A direct comparison was then carried out and the smallest impedance computed was selected. Simulations on the IEEE 14-bus test system were done to demonstrate and validate the proposed methodology. According to the results, the CPF method should be used to compute the parameters to set the back-up zone in the distance protection. When using the OPF algorithm, the voltage magnitude at all buses is close to the nominal value and the power flow in transmission lines is low, in comparison with the CPF. This result is obtained because of the constraints introduced in the OPF formulation. By setting the back-up zone with the calculated parameters, undesirable disconnection of transmission lines can be avoided and, therefore, the power system would be operable under stressful conditions.

Table 3. Comparison of obtained impedances

\begin{tabular}{cccc}
\hline \multicolumn{2}{c}{$\begin{array}{c}\text { Transmission } \\
\text { line to set }\end{array}$} & $\begin{array}{c}\text { Impedance to adjust } \\
\text { at relay using the CPF }\end{array}$ & $\begin{array}{c}\text { Impedance to adjust } \\
\text { at relay using the OPF }\end{array}$ \\
From bus & To bus & (p.u.) & (p.u.) \\
\hline 2 & 5 & 0.254188762 & 1.801277526 \\
1 & 2 & 0.067850151 & 0.661375661 \\
3 & 2 & 0.183981865 & 1.355203375 \\
3 & 4 & 0.238014223 & 1.17861727 \\
1 & 5 & 0.221694508 & 0.747638143 \\
5 & 4 & 0.147995052 & 1.067097214 \\
2 & 4 & 0.213754996 & 1.405681184 \\
5 & 2 & 0.163673145 & 1.630453752 \\
2 & 1 & 0.066952277 & 0.631648856 \\
2 & 3 & 0.190104265 & 1.443659089 \\
4 & 3 & 0.187783358 & 1.245488637 \\
5 & 1 & 0.146422899 & 1.454664342 \\
4 & 5 & 0.154566847 & 1.047850459 \\
4 & 2 & 0.139943546 & 1.292744107 \\
\hline
\end{tabular}


Table 4. Correct settings to avoid an undesirable trip of the distance protection

\begin{tabular}{ccc}
\hline $\begin{array}{c}\text { Transmission line } \\
\text { to set }\end{array}$ & $\begin{array}{c}\text { Impedance to set in the } \\
\text { relay at sending end }\end{array}$ \\
From bus & To bus & (p.u.) \\
\hline 2 & 5 & 0.254188762 \\
1 & 2 & 0.067850151 \\
3 & 2 & 0.183981865 \\
3 & 4 & 0.238014223 \\
1 & 5 & 0.221694508 \\
5 & 4 & 0.147995052 \\
2 & 4 & 0.213754996 \\
5 & 2 & 0.163673145 \\
2 & 1 & 0.066952277 \\
2 & 3 & 0.190104265 \\
4 & 3 & 0.187783358 \\
5 & 1 & 0.146422899 \\
4 & 5 & 0.154566847 \\
4 & 2 & 0.139943546 \\
\hline
\end{tabular}

Source: Authors, 2012.

\section{NOMENCLATURE}

$B_{i j} \quad i j$-th element of the bus susceptance matrix B

$b_{i j} \quad$ series susceptance of the branch $(i, j)$

$b_{i j}^{s h} \quad$ shunt susceptance of the branch $(i, j)$

$c \quad$ dimension of the tangent vector

CPF Continuation Power Flow scalar nonlinear objective function

$f(\cdot) \quad$ in the OPF formulation with $n$ unknowns

$g(\cdot) \quad m$-vector of equality constraints in the OPF formulation

$G_{i j} \quad i j$-th element of the bus conductance matrix $\mathrm{G}$

$G_{i j} \quad i j$-th element of the bus conductance matrix $\mathrm{G}$

$g_{i j} \quad$ series conductance of the branch $(i, j)$

$h(\cdot) \quad p$-vector of functional variables in the OPF formulation $h_{\text {max }} \quad$ upper bound of $h(\cdot)$

$h_{\text {min }} \quad$ lower bound of $h(\cdot)$

IEEE Institute of Electrical and Electronics Engineers

incidence matrix to obtain $\hat{I} x$, I $\quad$ which has $q$ number of simple bound constraints

$\bar{I}_{i j}^{1} \quad$ phasor of positive sequence current flowing from bus $i$ to bus $j$ $K_{d, i} \quad$ multiplier to designate the rate of load change at bus $i$

$K_{g, i} \quad$ multiplier to designate the rate of generation change at bus $i$

MLP Maximum Loading Point

$n_{1} \quad$ number of load buses

$n_{2} \quad$ number of generation buses

$N_{b} \quad$ number of buses in the power systems

$N_{l} \quad$ number of transmission lines in the power systems

OLTC On-Load Tap Changers

OPF Optimal Power Flow

p.u Per unit 
$P_{d, i} \quad$ active power load at bus $i$

$P_{d, i, 0} \quad$ active power load at bus $i$ in the base case

$\underline{P}_{g} \quad$ vector of active power generation

$P_{g, i} \quad$ active power generation at bus $i$

$P_{g, i, 0} \quad$ active power generation at bus $i$ in the base case

$P_{i} \quad$ active power injected at bus $i$

$P_{i j} \quad$ active power from the sending end in the transmission line $(i, j)$

$P_{j i} \quad$ active power from the receiving end in the transmission line $(i, j)$

$Q_{d, i} \quad$ reactive power load at bus $i$

$Q_{d, i, 0} \quad$ reactive power load at bus $i$ in the base case

$Q_{g} \quad$ vector of reactive power generation

$Q_{g, i} \quad$ reactive power generation at bus $i$

$Q_{i} \quad$ reactive power injected at bus $i$

$Q_{i j} \quad$ reactive power from the sending end in the transmission line $(i, j)$

$Q_{j i} \quad$ reactive power from the receiving end in the transmission line $(i, j)$

$\underline{r} \quad$ tangent vector

$\underline{t} \quad$ vector of transformer tap settings

$t_{i j} \quad$ tap setting of the branch $(i, j)$

$V C P \quad$ Voltage Collapse Point

v vector of voltage magnitudes

$v_{i} \quad$ bus voltage magnitude at bus $i$

$\bar{v}_{i}^{1}$ phasor of positive sequence voltage at the relay location

VSM Voltage Stability Margin

$\underline{x}$ vector of explicit decision variables

$\bar{Z}_{\text {relay }} \quad$ impedance seen by the relay

$\theta_{i} \quad$ bus voltage phase at bus $i$

$\lambda$ loading parameter

$\lambda_{\text {critical }}$ maximum loading parameter

represent the conjugated of the complex number

$\begin{array}{ll}\sigma & \text { scalar that designated the step size } \\ \underline{\theta} & \text { vector of voltage phases }\end{array}$

\section{REFERENCES}

Abba-Aliyu, S. (2009). Voltage Stability and Distance Protection Zone 3. Chalmers University of Technology, Gothenburg.
Alinezhad, M., \& Kamarposhti, M. A. (2009). Static voltage stability assessment considering the power system contingencies using continuation power flow method. Paper presented at the World Academy of Science, Engineering and Technology.

Chowdhury, B. H., \& Taylor, C. W. (2000). Voltage Stability Analysis: V-Q Power Flow Simulation Versus Dynamic Simulation. IEEE Transactions on Power Systems, 15(4), 1354-1359.

Gan, D., Thomas, R. J., \& Zimmerman, R. D. (2000). Stability-Constrained Optimal Power Flow. IEEE Transactions on Power Systems, 15(2), 535-540.

Gao, B., Morison, G. K., \& Kundur, P. (1992). Voltage Stability Evaluation Using Modal Analysis. IEEE Transactions on Power Systems, 17(4), 1529-1542.

Horowits, S. H., \& Phadke, A. G. (2006). Third Zone Revisited. IEEE Transactions on Power Delivery, 21(1), 23-29.

Johansson, S., \& Sjögren, F. (1995). Voltage Collapse on Power System. Chalmers University of Technology, Gothenburg.

Jonsson, M. (2001). Line Protection and Power System Collapse. Chalmers University of Technology, Gothenburg.

Jonsson, M. (2003). Protection Strategies to Mitigate Major Power System Breakdowns. Chalmers University of Technology, Gothenburg.

Jonsson, M., \& Daalder, J. E. (2003). An Adaptive Scheme to Prevent Undesirable Distance Protection Operation During Voltage Instability. IEEE Transactions on Power Delivery, 18(4), 1174-1180.

Kundur, P. (1994). Power System Stability and Control. New York: McGraw-Hill.

Milano, F. (2005). An open source power system analysis toolbox. IEEE Transactions on Power Delivery, 20(3), 1199-1206.

Momoh, J. A., \& Shu, J. S. (1999). Improved Interior Point Method for OPF Problems. IEEE Transactions on Power Systems, 14(3), 1114-1120.

Morison, G. K., Gao, B., \& Kundur, P. (1993). Voltage Stability Analysis Using Static and Dynamic Approaches. IEEE Transactions on Power Systems, 8(3), 1159-1171. 
Nagao, T., Tanaka, K., \& Takenaka, K. (1997). Development of Static and Simulation Programs for Voltage Stability Studies of Bulk Power System. IEEE Transactions on Power Systems, 12(1), 273-281.

Ohno, T., \& Imai, S. (2006, 29 October-1 November). The 1987 Tokyo Blackout. Paper presented at the Power Systems Conference and Exposition.

Overbye, T.J., Dobson, I., \& DeMarco, C.L. (1994). Q-V Curve Interpretation of Energy Measure for Voltage Security. IEEE Transactions on Power Systems, 9(1), 331-340.

Rajagopalan, C., Lesieutre, B., Sauer, P. W., \& Pai, M. A. (1992). Dynamic Aspects of Voltage/Power Characteristics. IEEE Transactions on Power Systems, 7(3), 990-1000.

Repo, S. (2001). On-Line Voltage Stability Assessment of Power System - An Approach of Black Box Modelling. Tampere University of Technology, Tampere.

Ruiz, M. (2006). Optimization of the MVAr Sources to Avoid Voltage Collapse. University of Costa Rica, San Jose.

Sancho, J., \& Vega, M. (2001). Reporte del apagón nacional del 6 de Marzo del 2001. San Jose: Instituto Costarricense de Electricidad.

Taylor, C. W. (1994). Power System Voltage Stability. New York: McGraw-Hill.

University of Washington. Power system test case archive. Retrieved Oct., 2010, from http:// www.ee.washington.edu/research/pstca/

Vaahedi, E., Tamby, J., Manssur, Y., Li, W., \& Sun, D. (1999). Large Scale Voltage Stability Constrained Optimal VAr Planning and Voltage Stability Applications Using Existing OPF/Optimal VAr Planning Tools. IEEE Transactions on Power Systems, 14(1), 65-74.
Van Cutsem, T., \& Vournas, C. (1998). Voltage Stability of Electric Power Systems: Kluwer Academic Publishers.

Venkataramana, A. (2006). Computational Techniques for Voltage Stability Assessment and Control. New York: Springer Science and Business Media.

Venkataramana, A., \& Christy, C. (1992). The Continuation Power Flow: A Tool for Steady State Voltage Stability Analysis. IEEE Transactions on Power Systems, 7(1), 416-423.

Yang, Z., Shi, D., \& X., D. (2006, October). Optimal Coordination of Distance Relays in Interconnected Power Systems. Paper presented at the International Conference on Power System Technology.

\section{ABOUT THE AUTHORS}

\section{Jairo Quirós Tortós}

Lecturer in the Department of Electric Machines and Power Systems, School of Electrical Engineering, University of Costa Rica and Ph.D. student at the University of Manchester. His main research interests are application of intelligent methods to power system restoration, and controlled islanding, power system monitoring, protection and control, voltage stability assessment, and power system dynamics. At the end of his Ph.D. studies, he will return to the University of Costa Rica as a Professor and Researcher.

E-mail: jairoquirostortos@iee.org

\section{Eddie A. Araya Padilla}

Cathedratic Professor in the Department of Electric Machines and Power Systems, School of Electrical Engineering, University of Costa Rica. His main research interests are nonlinear dynamics, FACTS devices and wind generation. E-mail: earaya@eie.ucr.ac.cr 
